\title{
ORAL CAVITY-DERIVED EXOSOMES AS PROMISING TOOL IN CHRONIC WOUND HEALING
}

\author{
Blanka Borowiec ${ }^{1,2}$, Marta Dyszkiewicz-Konwińska ${ }^{2,3}$, Greg Hutchings ${ }^{4}$, Joanna Budna-Tukan ${ }^{1}$
}

\begin{abstract}
Wound healing is an important physiological process aimed at maintaining the integrity of the skin after injury, accidentally or intentionally. Physiological wound healing involves three consecutive but overlapping phases, including hemostasis, proliferation and remodeling. Wound healing abnormalities, such as excessive wound healing (e.g. keloid) or chronic wounds (e.g. ulcers) impair normal physiological function. Many experimental studies have provided insight into wound healing. There are numerous methods that support wound healing, including popular hydrogels, vegetable oils, ultrasound and even treatment with maggots. Stem cell therapies are also very popular, but they are not safe in all cases due to having specific antibodies. In the following article, in addition to a brief overview of current healing therapies we will examine exosomal therapy, which, although new, seems to be very promising if only because of the high safety of use.
\end{abstract}

Running title: Exosomes in wound healing

Keywords: oral cavity, mucosa, exosomes, wound healing 


\section{Introduction}

Wound healing is an indispensable, as well as challenging process occurring in humans and animals. Its complexity is based on steps, sequentially occurring one after another [1]. During the whole process phases like hemostasis, inflammation, proliferation and regeneration take place. These cell-matrix and cell-cell interactions proceed in three overlapping phases: inflammation (0-3 days), cellular proliferation (3-12 days) and remodeling (3-6 months) [2]. Successful outcome of this mechanism requires complex interactions between different types of cells, cytokines, growth factors and extracellular matrix elements [3]. That is why, although highly controlled, wound healing is an anomaly-prone process. Wounds caused by burns, chronic diseases and postoperative injuries can be fatal in many cases because they can easily be colonized by bacteria and microbes which can be highly resistant [2]. Thus, $96 \%$ of the 300,000 recorded burn incidents, can be life-threatening or even mortal to patients due to frequent complications [4]. Factors such as obesity or congenital diseases raise the risk of wound infections and particularly of wound sepsis [5]. Healed wounds often turn into deep, hard to remove scars or, in the case of incorrect inflammatory reactions, chronic non-healing wounds [6]. The latter especially are a clinically and esthetically challenging problem. Frequency of chronic wounds correlates with age of patients and thus increasing average length of life in Western countries [7]. This brings a need to better characterize the process of wound healing in order to optimize methods of its treatment with the use of innovative bio-tools.

\section{Current wound healing approaches}

Currently, there are few known products applied in chronic wound healing. Among them we can mention: specialized wound dressings, including hydrogels and hydrocolloids, living skin substitutes, like allogenic bi-layered skin graft, composed of dermal fibroblasts enriched with bovine collagen type I and epidermal keratinocytes, growth factors and finally controversial hyperbaric oxygen therapies [8]. Hydrogels are three-dimensional polymeric networks. They are evaluated as highly biodegradable and biocompatible, applied effectively as wound healing systems and controlled drug transfer. The ideal material should mimic as many properties of human skin as possible. Hydrogels present high oxygen and molecular permeability, low interfacial tension, proper moisturizing and mechanical properties which resemble physiological soft tissue environment. Calcium ion-crosslinked nanofibrillated cellulose (NFC) hydrogels deserve special attention. The material's potential to maintain an appropriate moist environment for different types of wounds was shown in water retention tests. Also, the inert nature of hydrogels in conditions of cytokine se- cretion and free oxygen radicals was revealed by inflammatory response studies. Results highlight the great potential of ion-crosslinked NFC hydrogels for the development of advanced wound dressings, where further functionalization of the material could lead to improved properties towards the healing of specific wound types. The results clearly point to hydrogels as one of the best ways to heal wounds today, however they are not suitable for exudative wounds, where dressings must be changed frequently [9]. Since hydrogels are not absorbent, they may even prolong the healing process.

There are also natural products in traditional medicine, whose beneficial effects were discovered hundreds of years ago, but are also used nowadays. For instance, Rosmarinus officinalis oil reduces inflammation, enhances wound contraction, regeneration of granulation tissue, re-epithelialization, collagen deposition and even angiogenesis. The latter one and collagen deposition were detected in the treated wounds [10].

\section{Innovative wound healing therapies Therapy with a use of stem cells}

Stem cell therapies are also widely used. It is known that they can accelerate healing rate through reduction of scar contractures and inflammation. They also increase speed and quality of wounds' closure. Among their numerous advantages is ease of isolation and characterization. It is simple to extract them from their source, e.g. bone marrow or adipose tissue. Cell delivery methods are constantly being established, and additionally, very small amounts of them are required to start the cell culture of high survival rate. Unfortunately, stem cell application can elicit uncontrolled immunologic reaction to the treated patient. First of all, mechanisms of action should be identified, since they are still not well understood. The long-term safety profile should be investigated as there are currently no sources suggesting long-term stem cell treatment. Differentiation into many types of cells is somewhat limited, which means that they can only be applied to designated types of wounds [11].

\section{Therapy with the use of exosomes}

The solution to the problem above seems to be the use of molecules presenting high therapeutic safety, which are exosomes. They are shed by many cell types, both to extracellular matrix and biological fluids. They belong to the group of extracellular vesicles (EVs) and, similarly to the microvesicles located in this group, are separated from the surrounding environment by a lipid bilayer. Exosomes are vesicles of heterogeneous size (from 40 to 120 $\mathrm{nm}$ ), released by many types of cells, both into extracellular space and biological fluids [12]. When exosomes in reticulocytes were identified more than 30 years ago, it was believed that their main 
role was to selectively remove unwanted components from cells [13]. In recent years, however, they have gained great interest from researchers in the fields of medicine and biological sciences, which has led to the broadening of knowledge in this field. It is now known that they have the ability to transport biological molecules in an intact, active form, including proteins and nucleic acids, also to cells [14]. Thus, exosomes are seen as carrier elements that are part of the still unknown intercellular signaling pathways.

Although mechanisms of intercellular communication involving exosomes are not yet understood in detail, vesicles are already used in regenerative medicine and related sciences. Their regenerative potential has been used so far in repairing myocardial damage [15], parts of the brain affected by stroke [16], kidney defects caused by acute inflammation [17] or in removing liver fibrosis [18]. Regulation of cell growth by exosomes was used in turn to inhibit the growth of breast cancer cells in the mouse model [19] and in neurite proliferation in in vitro cultured rat neurons [20]. Furthermore, co-culture of mesenchymal stem cells (MSCs), secreting exosomes, with cancer cells initiated functional changes of both cell types, described as mutual cellular adaptation [21]. It is worth emphasizing that recently the relationship between exosomes and the process described above, which is wound regeneration and healing, has also been described. Literature sources state that this phenomenon is based on the ability of exosomes to initiate the process of angiogenesis, cell migration, their proliferation, as well as the process of epithelial reconstruction through the activation of certain signal pathways (AKT, ERK, STAT3, Wnt/ $\beta$-catenin), leading to an increase expression of growth factors $[22,23]$.

Unquestionable advantage of exosomes is their high therapeutic safety. They are unable to divide, thus there is no risk of uncontrolled proliferation, target cells and tissue outgrowth and predominately carcinogenesis. Similarly, there is no danger of unwanted immunological interactions, on the assumption that exosomes are derived from host's cells [24]. Additionally, taking into account bilayered structure of vesicles and capability of active particles' transport, there is a possibility to modify their composition with a use of biological engineering [25].

\section{Oral cavity as a promising source of therapeutic exosomes}

Thus, tissues with high potential for regeneration and self-renewal seem to be promising source of exosomes, favoring wound healing. Unquestionably, one of them is mucosa lining oral cavity. Physiologically, this region is constantly exposed to injury, however renewal and healing are very prompt. Oral cavity is easily accessible and displays unique characteristics such as fetal-like wound healing [26] and antibacterial properties $[27,28]$. The oral cavity has antibacterial properties as a part of the native immunity. Epithelial antimicrobial peptides ( $\beta$-defensin coming from oral epithelium) have been identified and examined [29]. Also osteoprotegerin and haptoglobin - peptides secreted by progenitor cells of lamina propria, reduce growth of several pathogens [27]. It is easy to notice that wounds appearing in this region are healed much faster than those affecting skin, carrying low risk of scars formation. It can be assumed that it is caused by smaller amount of blood vessels and immunological and profibrogenic mediators, comparing to skin. On the other hand, mucosa can be characterized by highly effective epithelial renewal and fast-rate proliferation of underlying fibroblasts [30]. All this can be attributed to the presence of cells with stemness features, enabling continuous restoration of tissues from the oral cavity [31]. Furthermore, salivary exosomes have been identified as highly informative nanovesicles. They have shown a pathway and machinery by which cancer-derived biomarkers can be ferried through the systemic circulation into the oral cavity [32]. This provides the basis for studies of interaction between saliva and oral mucosa and indicates a close relationship between these two elements.

\section{Future perspectives}

Several innovative wound care therapies are currently known. Negative pressure wound therapy (NPWT) uses controlled vacuum produced on the wound surface to improve healing. By moving gas molecules using suction, generated by vacuum system, an underpressure is created. This provides a semi-occlusive environment that promotes wound healing. Next, biosurgery is a therapeutic method of cleansing and stimulating the wound healing process, which uses the phenomenon of external digestion occurring in the larvae of the flies - Lucilia (Phaenicia) sericata. The post-embryonic stages of Phaenicia sericata fly are facultative parasites of birds and mammals, feeding on their living tissues in a process called myiasis. The development of the larvae lasts about 20 days, so the wounds in which flies lay their eggs must heal quickly $[33,34]$. As it turns out, sounds can also be used to heal wounds. Sonotherapy is a selective, but at the same time very effective method of cleaning acute and chronic wounds (ultrasonic-assisted wound debridement - UAW). Low-frequency ultrasound produces non-thermal effects in tissues - cavitation and acoustic flux. The acoustic stream in turn initiates unidirectional fluid movement in the ultrasonic field, stimulating the activity and migration of phagocytic cells, supporting the natural mechanisms of wound cleansing and proliferation [35]. Fluid mechanics can also be useful. Hydrosurgical wound cleansing (Versajet system) is a method combining aggressive and highly selective wound development with 
pulsed rinsing. It uses a small stream of fluid under high pressure and enormous speed (the so-called water knife), which cuts and removes tissues. This is possible thanks to the vacuum created simultaneously in the head of the tip, which sucks and removes cut tissue and impurities [36].

The above-mentioned methods are certainly effective, but there is often a number of contraindications for each of them. The idea of mucosal exosomes usage in wound healing seems to be promisingly safe, but obviously needs further laboratory studies. The knowledge describing how the parental cell produces exosomes and blend the potentially therapeutic ingredients, such as miRNA, into the exosome is crucial to optimize the production of effective therapeutic exosomes. In addition, a thorough understanding of the internalization of exosome by recipient cells is key to increasing the therapeutic efficacy of exosomes in gene therapy and drug delivery [37].

Based on information already presented isolation of exosomes derived from oral cavity cells could have beneficial influence on regeneration of chronic wounds. For this purpose in vitro culture of mucosal cells is necessary in order to isolate exosomes from their culture medium. The problem of poor availability of human tissues can be easily solved by, in fact, unlimited access to recovered at slaughter animal tissues. The best option seems to be working on pig (Sus scrofa f. domestica) model, greatly resembling human in the aspect of genome length, dentition, physiology of cardiovascular and digestive tract, anatomy and lastly morphology [38]. The most important step, before clinical testing, requires in-depth analysis of transcriptomic and proteomic profile of oral cavity-derived exosomes in reference to regeneration. This knowledge would reveal the intercellular mechanisms of action and determine exosomal elements playing a vital role in the process of healing.

\section{Conclusions}

Although there are many wound healing techniques that have been used for a long time, scientists continually improve them or search for innovative solutions. The superior aim of the studies in the field of chronic wound healing is finding new cellular markers assuring effective regeneration. Secretome of stem-like mucosal cells, in the form of exosomes, seems to be very promising tool for regenerative medicine, excluding problems related to live cell therapy. Using of animal models closely related to human may be helpful in understanding yet undiscovered mechanisms of wound healing. Revealing the physiological role of an entirely new exosome-mediated cell-cell communication can further improve clinical wound care therapy. However, it is of predominant importance to describe role and significance of exosomal components in the process of wound healing, what would estimate their prospective share in innovative regenerative medicine.

\section{Ethical approval}

The conducted research is not related to either human or animal use.

\section{Corresponding author}

Joanna Budna-Tukan, Department of Histology and Embryology, Poznan University of Medical Sciences, 6 Święcickiego St., 60781 Poznań, Poland, Tel./Fax: +48 61 8546428, e-mail: jbudna@ ump.edu.pl.

\section{Conflict of interest statement}

The authors declare they have no conflict of interest.

\section{References}

1. Wang P-H, Huang B-S, Horng H-C, Yeh C-C, Chen Y-J. Wound healing. J Chinese Med Assoc. 2018;81:94-101; DOI:10.1016/j.jcma.2017.11.002.

2. Üstündağ Okur N, Hökenek N, Okur ME, Ayla Ş, Yoltaş A, Siafaka PI, et al. An alternative approach to wound healing field; new composite fil$\mathrm{ms}$ from natural polymers for mupirocin dermal delivery. Saudi Pharm J SPJ Off Publ Saudi Pharm Soc. 2019;27:738-52; DOI:10.1016/j. jsps.2019.04.010.

3. Reinke JM, Sorg H. Wound Repair and Regeneration. Eur Surg Res. 2012;49:35-43; DOI:10.1159/000339613.

4. Khalid A, Khan R, Ul-Islam M, Khan T, Wahid F. Bacterial cellulose-zinc oxide nanocomposites as a novel dressing system for burn wounds. Carbohydr Polym. 2017;164:214-21; DOI:10.1016/j.carbpol.2017.01.061.

5. Cambiaso-Daniel J, Gallagher JJ, Norbury WB, Finnerty CC, Culnan DM. Treatment of Infection in Burn Patients. Total Burn Care. 2018:93-113. e4; DOI:10.1016/B978-0-323-47661-4.00011-3.

6. Zhao R, Liang H, Clarke E, Jackson C, Xue M. Inflammation in Chronic Wounds. Int J Mol Sci. 2016;17DOI:10.3390/ijms17122085.

7. Potempa M, Jonczyk P, Janerka M, Kucharzewski M, Krawczyk-Krupka A. Determinants and epidemiology of chronic wounds. Leczenie Ran. 2014;11:43-50; DOI:10.15374/LR2014007.

8. You H-J, Han S-K. Cell therapy for wound healing. J Korean Med Sci. 2014;29:311-9; DOI:10.3346/jkms.2014.29.3.311.

9. Skórkowska-Telichowska K, Czemplik M, Kulma A, Szopa J. The local treatment and available dressings designed for chronic wounds. J Am Acad Dermatol. 2013;68:e117-26; DOI:10.1016/j.jaad.2011.06.028.

10. Abu-Al-Basal MA. Healing potential of Rosmarinus officinalis L. on full-thickness excision cutaneous wounds in alloxan-induced-diabetic BALB/c mice. J Ethnopharmacol. 2010;131:443-50; DOI:10.1016/j. jep.2010.07.007.

11. Otero-Viñas M, Falanga V. Mesenchymal Stem Cells in Chronic Wounds: The Spectrum from Basic to Advanced Therapy. Adv Wound Care. 2016;5:149-63; DOI:10.1089/wound.2015.0627.

12. Cobelli NJ, Leong DJ, Sun HB. Exosomes: biology, therapeutic potential, and emerging role in musculoskeletal repair and regeneration. Ann N Y Acad Sci. 2017;1410:57-67; DOI:10.1111/nyas.13469.

13. Johnstone RM, Adam M, Hammond JR, Orr L, Turbide C. Vesicle formation during reticulocyte maturation. Association of plasma membrane activities with released vesicles (exosomes). J Biol Chem. 1987;262:9412-20.

14. H Rashed M, Bayraktar E, K Helal G, Abd-Ellah MF, Amero P, Chavez-Reyes A, et al. Exosomes: From Garbage Bins to Promising Therapeutic Targets. Int J Mol Sci. 2017;18:538; DOI:10.3390/ijms18030538.

15. Lai RC, Arslan F, Lee MM, Sze NSK, Choo A, Chen TS, et al. Exosome secreted by MSC reduces myocardial ischemia/reperfusion injury. Stem Cell Res. 2010;4:214-22; DOI:10.1016/j.scr.2009.12.003.

16. Doeppner TR, Herz J, Görgens A, Schlechter J, Ludwig A-K, Radtke S, et al. Extracellular Vesicles Improve Post-Stroke Neuroregeneration and Prevent Postischemic Immunosuppression. Stem Cells Transl Med. 2015;4:1131-43; DOI:10.5966/sctm.2015-0078.

17. Bruno S, Grange C, Deregibus MC, Calogero RA, Saviozzi S, Collino F, et al. Mesenchymal stem cell-derived microvesicles protect against acute tubular injury. J Am Soc Nephrol. 2009;20:1053-67; DOI:10.1681/ ASN.2008070798.

18. Li T, Yan Y, Wang B, Qian H, Zhang X, Shen L, et al. Exosomes Derived from Human Umbilical Cord Mesenchymal Stem Cells Alleviate Liver Fibrosis. Stem Cells Dev. 2013;22:845-54; DOI:10.1089/scd.2012.0395.

19. Lee J-K, Park S-R, Jung B-K, Jeon Y-K, Lee Y-S, Kim M-K, et al. Exosomes derived from mesenchymal stem cells suppress angiogenesis by 
down-regulating VEGF expression in breast cancer cells. PLoS One. 2013;8:e84256; DOI:10.1371/journal.pone.0084256.

20. Xin H, Li Y, Buller B, Katakowski M, Zhang Y, Wang X, et al. Exosome-mediated transfer of miR-133b from multipotent mesenchymal stromal cells to neural cells contributes to neurite outgrowth. Stem Cells. 2012;30:1556-64; DOI:10.1002/stem.1129.

21. Yang Y, Otte A, Hass R. Human mesenchymal stroma/stem cells exchange membrane proteins and alter functionality during interaction with different tumor cell lines. Stem Cells Dev. 2015;24:1205-22; DOI:10.1089/ scd.2014.0413.

22. Goodarzi P, Larijani B, Alavi-Moghadam S, Tayanloo-Beik A, Mohamadi-Jahani F, Ranjbaran N, et al. Mesenchymal Stem Cells-Derived Exosomes for Wound Regeneration. Adv. Exp. Med. Biol., vol. 1119, 2018, p. 119-31DOI:10.1007/5584_2018_251.

23. Rani S, Ritter T. The Exosome - A Naturally Secreted Nanoparticle and its Application to Wound Healing. Adv Mater. 2016;28:5542-52; DOI:10.1002/adma.201504009.

24. Sarko DK, McKinney CE. Exosomes: Origins and Therapeutic Potential for Neurodegenerative Disease. Front Neurosci. 2017;11:82; DOI:10.3389/fnins.2017.00082.

25. Xitong D, Xiaorong Z. Targeted therapeutic delivery using engineered exosomes and its applications in cardiovascular diseases. Gene. 2016;575:377-84; DOI:10.1016/j.gene.2015.08.067.

26. Mak K, Manji A, Gallant-Behm C, Wiebe C, Hart DA, Larjava H, et al Scarless healing of oral mucosa is characterized by faster resolution of inflammation and control of myofibroblast action compared to skin wounds in the red Duroc pig model. J Dermatol Sci. 2009;56:168-80; DOI:10.1016/j.jdermsci.2009.09.005.

27. Board-Davies E, Moses R, Sloan A, Stephens P, Davies LC. Oral Mucosal Lamina Propria-Progenitor Cells Exert Antibacterial Properties via the Secretion of Osteoprotegerin and Haptoglobin. Stem Cells Transl Med. 2015;4:1283-93; DOI:10.5966/sctm.2015-0043.

28. Khurshid Z, Naseem M, Sheikh Z, Najeeb S, Shahab S, Zafar MS. Oral antimicrobial peptides: Types and role in the oral cavity. Saudi Pharm J. 2016;24:515-24; DOI:10.1016/j.jsps.2015.02.015.

29. Hans M, Madaan Hans V. Epithelial Antimicrobial Peptides: Guardian of the Oral Cavity. Int J Pept. 2014;2014:1-13; DOI:10.1155/2014/370297.

30. Morton LM, Phillips TJ. Wound healing and treating wounds. J Am Acad Dermatol. 2016;74:589-605; DOI:10.1016/j.jaad.2015.08.068.

31. Leavitt T, Hu MS, Marshall CD, Barnes LA, Lorenz HP, Longaker MT. Scarless wound healing: finding the right cells and signals. Cell Tissue Res. 2016;365:483-93; DOI:10.1007/s00441-016-2424-8.

32. Cheng J, Nonaka T, Wong DTW. Salivary Exosomes as Nanocarriers for Cancer Biomarker Delivery. Mater (Basel, Switzerland) 2019;12DOI:10.3390/ma12040654.

33. Gupta A. A Review of the Use of Maggots in Wound Therapy. Ann Plast Surg. 2008;60:224-7; DOI:10.1097/SAP.0b013e318053eb5e.

34. Choudhary V, Choudhary M, Pandey S, Chauhan VD, Hasnani JJ. Maggot debridement therapy as primary tool to treat chronic wound of animals. Vet World. 2016;9:403-9; DOI:10.14202/vetworld.2016.403-409.

35. Dissemond J, Fitz G, Goos M. Konditionierung chronischer Wunden mittels Ultraschall. Der Hautarzt. 2003;54:524-9; DOI:10.1007/ s00105-003-0520-7.

36. Wong WL, Wong She R, Mathy JA. Rhinophyma treatment using Versajet hydrosurgery. ANZ J Surg. 2017;87:E331-2; DOI:10.1111/ans.13189.

37. Cobelli NJ, Leong DJ, Sun HB. Exosomes: biology, therapeutic potential, and emerging role in musculoskeletal repair and regeneration. Ann N Y Acad Sci. 2017;1410:57-67; DOI:10.1111/nyas.13469.

38. Sullivan TP, Eaglstein WH, Davis SC, Mertz P. The pig as a model for human wound healing. Wound Repair Regen. 2001;9:66-76. 